\title{
CARACTERÍSTICAS DE LA MUERTE DE MUJERES POR VIOLENCIA SEGÚN LAS NECROPSIAS REALIZADAS EN LA MORGUE DEL CALLAO
}

\author{
Kelly M. Casana-Jara(1),2,a \\ ${ }^{1}$ Instituto de Medicina Legal y Ciencias Forenses, Lima, Perú. \\ ${ }^{2}$ Escuela de Posgrado, Universidad Privada Norbert Wiener, Lima, Perú. \\ ${ }^{a}$ Médica especialista en Medicina Legal, doctora en Gestión Pública y Gobernabilidad.
}

\section{RESUMEN}

El objetivo fue describir las características de la muerte de mujeres por violencia según las necropsias realizadas en la morgue del Callao desde el 2016 al 2018. Se revisaron los registros forenses de 83 mujeres y se encontró que la muerte de mujeres por violencia ocurrió con mayor frecuencia en la etapa adulta, el suceso de tránsito fue el agente causante más implicado, la lesión mortal se ubicó mayormente en el segmento cabeza, se realizaron más levantamientos de cadáveres en la vía pública y el distrito con más casos fue el Callao. Es importante que las autoridades se comprometan a crear, instalar y seguir un plan de acción para prevenir la muerte de mujeres por violencia en el Callao.

Palabras clave: Autopsia; Causa de Muerte; Violencia; Violencia contra la Mujer; Cadáver; Accidente de Tránsito; Lesiones Craneoencefálicas; Lesiones Múltiples; Homicidio (fuente: DeCS BIREME).

\section{CHARACTERISTICS OF WOMEN'S DEATH BY VIOLENCE ACCORDING TO NECROPSIES CARRIED OUT IN THE CALLAO MORGUE}

\begin{abstract}
The objective was to describe the characteristics of women's deaths by violence according to autopsies performed at the Callao morgue from 2016 to 2018. The forensic records of 83 women were reviewed and it was found that women's deaths by violence occurred most often in adulthood. Traffic accidents were found to be the most common cause. The most frequent location of the fatal injury was in the head segment. Most of the corpse removal took place on the public road. The district with the most cases was Callao. It is important that the authorities commit to creating, installing and following an action plan to prevent women's deaths by violence in Callao.
\end{abstract}

Keywords: Autopsy; Cause of Death; Violence; Violence against Women; Cadaver; Traffic accident; Craniocerebral Trauma; Multiple Trauma; Homicide (source: MeSH NLM).

\section{INTRODUCCIÓN}

La muerte violenta o de causa no natural se considera a aquella cuyo origen es traumático (mecánico, físico, asfíctico, tóxico, térmico, físico, etc.) y cuya etiología medicolegal corresponde a un homicidio, suicidio o accidente ${ }^{(1)}$. En el mundo, los hechos violentos que no necesariamente implican muerte se ubican en los primeros lugares del perfil epidemiológico en todos los estratos sociales.

La Organización Mundial de la Salud (OMS) define violencia como «el uso intencional de fuerza física o poder, bajo amenaza o efectiva, contra uno mismo, otra persona o un grupo o comunidad, que cause o tenga muchas probabilidades de ocasionar lesiones, muerte, daño psicológico, trastorno del desarrollo o deprivación» ${ }^{(2)}$. La OMS también reporta que en un día mueren más de 15000 personas a causa de un hecho violento, como sucesos de tránsito (23\%), otros (21\%) (asfixia, mordeduras de animales venenosos, hipo e hipertermia, y desastres naturales), suicidio (15\%), homicidio (11\%), caídas (8\%), ahogamiento (7\%), quemaduras $(6 \%)$, intoxicaciones $(6 \%)$, guerra $(3 \%)^{(3)}$.

En el Perú las formas de violencia más utilizadas contra las mujeres son los golpes, la asfixia, el uso de cuchillos, el uso de armas de fuego, aplastamiento, decapitación, quemaduras, 
entre otras ${ }^{(4)}$. Todo acto violento constituye un problema de salud pública pues ocasiona lesión física, discapacidad, secuelas, disminución de la calidad de vida y finalmente la muerte ${ }^{(5)}$.

La necropsia de ley se realiza por disposición de la autoridad encargada de la investigación (fiscal, juez), es un procedimiento médico, técnico y científico que permite establecer la causa, el tiempo, el agente causante, la manera y los mecanismos de la muerte, así como la identificación del occiso, lo que aportará evidencias que contribuyan a la adecuada administración de justicia. Los casos médicolegales a los cuales se les realiza la necropsia de ley son las muertes violentas (no naturales, accidentes, suicidios y homicidios), las muertes sospechosas (aquellas que pueden ser violentas), las muertes súbitas e inesperadas, las muertes sin asistencia médica y las muertes en prisión ${ }^{(6)}$.

Es importante conocer las características de la muerte violenta en las mujeres desde el punto de vista médico legal, no solo con el enfoque de género, ni de la figura jurídica del feminicidio, sino enmarcada a una serie de casos de muertes violentas, a fin de conocer los agentes causantes, los segmentos corporales más vulnerables, la gestión del trauma, entre otros. Esto ayudará a identificar este problema en la provincia constitucional del Callao.

En ese sentido el objetivo de esta investigación es describir las características de la muerte de mujeres por violencia a quienes se les realizó la necropsia de ley en la morgue del Callao desde el 2016 al 2018.

\section{EL ESTUDIO}

Se realizó un estudio de serie de casos. La primera información se obtuvo del reporte estadístico del sistema informático DICETA (software de la morgue del Callao), en el cual se registran los datos generales del occiso, la autoridad solicitante, los hallazgos en la necropsia, exámenes auxiliares, la causa de la muerte y el agente causante. Se identificaron 83 casos de mujeres que tuvieron una muerte violenta, a las cuales se les realizaron las necropsias de ley solicitadas por las autoridades encargadas de la investigación, entre enero del 2016 y diciembre del 2018 en la morgue de la provincia constitucional del Callao. Luego se revisaron los protocolos de necropsia de los casos seleccionados. Se incluyeron los reportes de los protocolos de necropsia correspondientes a mujeres de 12 o más años con tipo de muerte violenta, y se excluyeron aquellos que no contaban con la causa de muerte o el agente causante definitivo.

Se analizaron las variables demográficas por grupos etarios según la clasificación del Programa de Atención Integral del Ministerio de Salud (7): adolescente (12 a 17años), joven (18 a 29 años), adulto (30 a 59 años) y adulto mayor (60 años y más); por distritos de la provincia constitucional del Callao: Bellavista, Callao, Carmen de la Legua Reynoso, La Per-

\section{MENSAJES CLAVE}

Motivación para realizar el estudio: Las muertes de mujeres por violencia se siguen incrementado y no todas corresponden a la violencia enfocada en el género, pues con características similares pueden constituir muertes accidentales.

Principales hallazgos: Como causa de muerte más frecuente se encontró al suceso de tránsito y al segmento cabeza como el área anatómica con más daño. En la mayoría de ellos la vía pública fue el escenario primario de la muerte.

Implicancias: Es importante establecer la causa de la muerte para diferenciar las implicancias legales de lesiones y las ligadas a feminicidio.

la, La Punta, Mi Perú y Ventanilla; por el lugar de ocurrencia según el acta de levantamiento de cadáver (documento medicolegal realizado por el médico legista con presencia fiscal, está incluido en el protocolo de necropsia): casa, hospital, hotel, terreno descampado, río, vía pública; por el agente causante que produjo la muerte violenta: agente contuso, elemento constrictor cervical, físico, líquido, proyectil de arma de fuego, punta o filo, suceso de tránsito; y según la anatomía topográfica, es decir el segmento dónde se ubicó la lesión que produjo la muerte: abdomen, cabeza, cuello, extremidades, múltiples (tres a más segmentos corporales), toracoabdominal y tórax.

Esta investigación es de orden científico, con fines estadísticos y epidemiológicos, se salvaguarda la identificación y reserva de los casos. La información encontrada en la base de datos corresponde al sistema de vigilancia del Plan Operativo Institucional del Instituto de Medicina Legal y del Observatorio de Criminalidad del Ministerio Público.

Se obtuvieron los permisos correspondientes del Instituto de Medicina Legal y Ciencias Forenses para acceder a la base de datos y publicar posteriormente los hallazgos. Los datos obtenidos se recolectaron en una plantilla (ficha de recolección de datos) diseñada por el autor, luego se procesó con el programa Microsoft Excel, donde se calcularon frecuencias y porcentajes.

\section{HALLAZGOS}

Al revisar el sistema informático de la morgue del Callao, en el 2016 se realizaron 571 necropsias, de las cuales 20 (3,5\%) correspondieron a muertes de mujeres por violencia; en el 2017, 520 necropsias, de las cuales 37 (7,0\%) correspondieron a muertes de mujeres por violencia; y en el 2018 se reali- 
zaron 579 necropsias, de las cuales 26 (4,5\%) correspondieron a muertes de mujeres por violencia, ello así, contamos un total de 83 casos. El grupo etario de 30-59 años fue el más frecuente con 35 casos (42,2\%) (Tabla 1$)$.

Los agentes que ocasionaron la muerte de las mujeres fueron, en primer lugar, los sucesos de tránsito que produjeron 26 muertes $(31,3 \%)$, seguido por el agente contuso que ocasionó 21 muertes (25,3\%). De acuerdo con la anatomía topográfica se identificó que la cabeza constituyó el segmento corporal cuya lesión ocasionó directamente la muerte en $27(32,5 \%)$ de las mujeres. Conforme a la revisión del acta

Tabla 1. Características de la muerte de mujeres por violencia, según las necropsias realizadas en la morgue del Callao, Perú, 2016-2018

\begin{tabular}{lc}
\hline Características & $\mathbf{n}(\%)$ \\
\hline Casos por año ${ }^{\mathrm{a}}$ & \\
2017 & $37 / 579(7,0)$ \\
2018 & $26 / 520(4,5)$ \\
2016 & $20 / 571(3,5)$ \\
Grupo etario (años) & \\
$12-17$ & $6(7,2)$ \\
$18-29$ & $15(18,1)$ \\
$30-59$ & $35(42,2)$ \\
60 y más & $27(32,5)$ \\
Agente causante & \\
Suceso de tránsito & $26(31,3)$ \\
Agente contuso & $21(25,3)$ \\
Proyectil de arma de fuego & $15(18,1)$ \\
Elemento constrictor cervical & $12(14,5)$ \\
Punta o filo & $5(6,0)$ \\
Físico ${ }^{\text {b }}$ & $3(3,6)$ \\
Líquido ${ }^{\text {c }}$ & $1(1,2)$ \\
Anatomía topográfica & \\
Cabeza & $27(32,5)$ \\
Múltiples ${ }^{\text {d }}$ & $23(27,7)$ \\
Cuello & $17(20,5)$ \\
Tórax & $8(9,6)$ \\
Toracoabdominal & $5(6,0)$ \\
Extremidades & $2(2,4)$ \\
Abdomen & $1(1,2)$ \\
Lugar de ocurrencia & \\
Vía pública & $34(41,0)$ \\
Hospital & $26(31,3)$ \\
Casa & $19(22,9)$ \\
Hotel & $2,2)$ \\
\hline
\end{tabular}

a El denominador corresponde al número de necropsias por año; ${ }^{\mathrm{b}}$ Fuego directo, corriente eléctrica; ${ }^{\mathrm{c}}$ agua, ${ }^{\mathrm{d}}$ tres o más segmentos corporales de levantamiento de cadáver, el lugar de ocurrencia más frecuente de estas muertes fue la vía pública con 34 casos (41,0\%), seguidos por el hospital con 26 casos (31,3\%). En cuanto a la ubicación geográfica por distritos se halló que en el Callao se presentaron 40 casos $(48,2 \%)$, seguido por Bellavista con 25 (30,1\%), Ventanilla con $11(13,3 \%)$, Carmen de la Legua Reynoso con $4(4,8 \%)$ y finalmente el distrito de La Perla con $3(3,6 \%)$.

\section{DISCUSIÓN}

La mayor cantidad de casos durante los tres años se presentó en el 2017, luego de casi tres meses del cese del estado de emergencia en el Callao ${ }^{(8)}$; con esta información se evidencia el estado dinámico que tiene la violencia y su relación con la muerte.

El grupo etario más frecuente fue el de 30-59 años; esto se relaciona con investigaciones internacionales de muertes violentas en general, con grupos de edades que oscilan entre los 18 a 44 años ${ }^{(9)}$, entre los 15 a 34 años ${ }^{(10)}$, entre los 20 a 40 años ${ }^{(11)}$ y con lo reportado por el Ministerio Público a nivel nacional ${ }^{(12)}$.

El agente causante que produjo directamente la muerte fue el suceso de tránsito, lo que coincide con una investigación en Colombia durante 1997-2003 ${ }^{(13)}$, y con lo encontrado en un estudio de Tijuana (México), donde el 55\% de muertes ocurrieron por atropellamiento ${ }^{(14)}$, esto difiere de lo reportado en Argentina, donde el suceso de tránsito fue la segunda causa de muerte ${ }^{(15)}$. Esta información varía entre países como Argentina, Brasil y Colombia, este último presenta un incremento exponencial de esta causa de muerte ${ }^{(16)}$.

Luego tenemos al agente contuso (objeto de borde romo) como causante, lo que coincide con lo descrito por Arias, et al. en un estudio sobre muertes violentas en la región Callao ${ }^{(17)}, \mathrm{y}$ que guarda relación con lo hallado por Serfaty, et al. en un estudio realizado en Argentina que reporta las muertes violentas en adolescentes y jóvenes ${ }^{(15)}$. Otras lesiones fueron ocasionadas por proyectiles disparados por armas de fuego. Este hallazgo confirma su presencia ilegal en las calles, lo que ha sido descrito por Meneses ${ }^{(18)}$ en México y por Arias, et al. en la región Callao ${ }^{(17)}$. Asimismo, también ha sido reportado por González, et al. en una investigación mexicana donde las armas de fuego estuvieron presentes ${ }^{(19)}$. En otro estudio colombiano las muertes violentas con trauma fueron ocasionadas por los proyectiles disparados por un arma de fuego, contusiones y heridas por arma cortopunzante ${ }^{(20)}$.

Durante el examen externo e interno del cadáver se identificó al segmento cabeza como el de mayor frecuencia de daño; hallazgos similares fueron encontrados en México en una investigación de muertes violentas por proyectiles de arma de fuego ${ }^{(21)}$. Al revisar el acta de levantamiento de cadáver, el lugar de ocurrencia de estas muertes fueron escenarios primarios (donde ocurrió la muerte) y escenarios 
secundarios (lugar donde no se produjo la lesión, pero sí ocurrió la muerte) entre ellos destaca la vía pública, seguido por el hospital.

Entre el 2014 y el 2015, en el Instituto de Medicina Legal de Cuiabá (Brasil) se revisaron 486 informes de necropsias de causa violenta. Se halló que el $68,1 \%$ de las muertes eran de personas entre 20 y 49 años, información que se relaciona con los datos encontrados en el Callao. También se identificó que el $64,4 \%$ de las muertes ocurrieron en la vía pública, dato relevante que es cercano a hallazgo en esta investigación. Se describe que la causa de la muerte más frecuente $(74,4 \%)$ se debió a lesión cerebral traumática y choque hipovolémico, en esta investigación en el segmento cabeza se identificó mayor daño y lesión ${ }^{(22)}$.

A pesar de los múltiples intentos de reducción de la violencia, de los estados de emergencia declarados por el Estado peruano, el Callao sigue siendo una provincia donde se deben redoblar esfuerzos en seguridad ciudadana, tal como se refleja en estos hallazgos; resultados similares han sido descritos en un estudio realizado con datos del 2003 al 2012, en la misma provincia ${ }^{(17)}$.

Se deben destacar las fortalezas del estudio, como laborar en la sede institucional encargada de las necropsias y la experiencia en el campo forense. Entre las limitaciones se

\section{REFERENCIAS BIBLIOGRÁFICAS}

1. Palomo Rando JL, Ramos Medina V, De La Cruz Mera E, López AM. Diagnóstico del origen y la causa de la muerte después de la autopsia médico-legal (Parte I). Cuad med forense [Internet]. 2010 [citado el 9 de enero de 2020]; 16(4):217-29. Disponible en: http://scielo.isciii.es/ scielo.php?script=sci_arttext\&pid=S1135-76062010000300005\&lng=es.

2. World Health Organization. World report on violence and health [Internet]. Geneva: WHO; 2002 [citado el 7 de enero de 2020]. Disponible en: https://apps.who.int/iris/bitstream/handle/10665/42495/9241545615_ eng.pdf?sequence $=1$.

3. World Health Organization. Injuries and violence, the facts. Ginebra: Department of Violence and Injury Prevention and Disability, WHO; 2010 [citado el 7 de enero de 2020]. Disponible en: https://apps.who.int/ iris/bitstream/handle/10665/44288/9789241599375_eng.pdf.

4. Garmendia F. La violencia en el Perú 2015. An Fac med [Internet]. 2016 [citado el 7 de enero del 2020]; 77 (2): 153-161. Disponible en: http:// dx.doi.org/10.15381/anales.v77i2.11838.

5. López M, Blanco J, Hijar M. La violencia y sus repercusiones en la salud; reflexiones teóricas y magnitud del problema en México. Salud Publica Méx [Internet]. 1997 [citado 16 de enero de 2020]; 39 (6): 565-572. Disponible en: http://saludpublica.mx/index.php/spm/article/view/6046/6951.

6. Di Maio V, Dana S. Manual de patologia forense. Austin, Texas. M.D. Press; 1998.

7. Modifican documento aprobado por RM No 729-2003-SA/DM en la clasificación de los Grupos Objetivo para los Programas de Atención Integral. Resolución Ministerial No 538-2009/MINSA [Internet]. Diario El Peruano. 19 agosto 2009 [citado el 7 de enero de 2020]. Disponible en: https://elperuano.pe/NormasElperuano/2009/08/19/385059-4.html.

8. Radio Programas del Perú [Internet]. Lima: RPP; 2016 [citado el 9 de enero de 2020]. El estado de emergencia en el Callao culminó esta medianoche; Disponible en: https://rpp.pe/peru/callao/el-estado-de-emergencia-en-el-callao-culmino-esta-medianoche-noticia-1002563. debe reconocer la no incorporación de casos sin diagnóstico definitivo, y de aquellas muertes ocurridas en el Callao pero que, por jurisdicción policial o fiscal fueron trasladas a la Morgue Central de Lima.

En conclusión, de los 83 casos de muerte de mujeres por violencia, estas ocurrieron mayormente en la etapa adulta, el suceso de tránsito representó el agente causante de mayor frecuencia, la lesión mortal que ocasionó la mayoría de estos casos se ubicó en el segmento cabeza, conforme al lugar de ocurrencia se presentaron más casos en la vía pública y finalmente el Callao fue el distrito con el mayor número de casos. Frente a ello se plantea contar con la participación de las autoridades nacionales para crear medios de gestión de políticas públicas integradas; contar con autoridades locales, incluyendo a los sectores fiscales y medicolegales, para crear, instalar y seguir un plan de acción para prevenir la violencia, que incluya un cronograma de actividades jurídico-penales, educativas, de salud y de bienestar social.

Contribuciones de los autores: KMCJ concibió, diseñó y redactó el presente artículo; y recolectó, analizó e interpretó los datos obtenidos.

Financiamiento: Autofinanciado.

Conflictos de interés: No se presentaron conflictos de intereses.

9. Rozo Lesmes P. Caracterización de la violencia homicida en mujeres en Bogotá 2000-2006 [Tesis de maestría]. Bogotá: Facultad de Medicina, Universidad Nacional de Colombia; 2007. Disponible en: http://bdigital. unal.edu.co/654/.

10. Chamizo H. Las muertes violentas en Costa Rica y sus inequidades geográficas. Población y Salud en Mesoamérica [Internet]. 2013 [citado el16 de enero de 2020]; 11(1):1-23. Disponible en: https://www.redalyc. org/articulo.oa?id=44628565006.

11. Bandala M, Melo G, Aguirre A. Prevalencia de muertes violentas en el Distrito Judicial X de Veracruz, México. Rev Mex Med Forense [Internet]. 2018 [citado el 9 de enero de 2020]; 3 (1): 19-26. Disponible en: https:// www.medigraphic.com/cgi-bin/new/resumen.cgi?IDARTICULO=88225.

12. Ministerio Público. Homicidio y feminicidio en el Perú Setiembre 2008 - Junio 2009 [Internet]. Lima: Observatorio de Criminalidad del Ministerio Público, MINPU; 2009 [citado el 16 de enero de 2020]. Disponible en: https://portal.mpfn.gob.pe/boletininformativo/infoestadfeminicidio

13. Sánchez R, Tejada P, Martínez J. Comportamiento de las Muertes Violentas en Bogotá, 1997-2003. Rev Salud pública [Internet]. 2005 [citado el 9 de enero de 2020]; 7(3):254-67. Disponible en: http://www.scielo.org. co/scielo.php?script=sci_arttext\&pid=S0124-00642005000300002\&1ng=en\&tlng=.

14. Molina A, Zonana A, Flores D, Martínez A, Quiroz M. Muertes violentas en Tijuana, Baja California, México. Salud Pública Méx [Internet]. 2008 [citado el 9 de enero de 2020]; 50 (2):105-6. Disponible en: http://saludpublica.mx/index.php/spm/article/view/6807/8564.

15. Serfaty E, Foglia V, Masaútis A, Negri G. Mortalidad por causas violentas en adolescentes y jóvenes de 10-24 años Argentina 1991 - 2000. Rev. Vertex. [Internet]. 2003 [citado el 9 de enero de 2020]; 14(2): 40-48. Disponible en: http://www.epidemiologia.anm.edu.ar/wp-content/uploads/2017/12/ Mortalidad_Causas_Violentas_2003.pdf.

16. Cardona D, Peláez E, Aidar T, Ribotta B, Álvarez M. Mortalidad por causas externas en tres ciudades latinoamericanas: Córdoba (Argentina), 
Campinas (Brasil) y Medellín (Colombia), 1980-2005. R bras Est Pop [Internet]. 2008 [citado el 9 de enero de 2020]; 25 (2): 335-352. Disponible en: https://doi.org/10.1590/S0102-30982008000200009.

17. Arias $\mathrm{M}$, Gutiérrez C. Patrón espacial y temporal de las muertes violentas por homicidios en la región Callao y su distribución según variables sociodemográficas, 2003 - 2012. Rev. Peruana de Epidemiología [Internet]. 2014. [citado el 11 de enero de 2020]; 18 (1): 1-5. Disponible en: http://ateneo.unmsm.edu.pe/bitstream/handle/123456789/3320/ rev_peru_epidemiol02v18n1_2014.pdf?sequence=1\&isAllowed=y.

18. Meneses R. Detalles de una muerte violenta: víctimas y circunstancias del homicidio por arma de fuego en el Distrito Federal (2000-2010). Andamios [Internet]. 2013 [citado el 9 de enero de 2020]; 10(23): 257-290. Disponible en: http://www.scielo.org.mx/scielo.php?script=sci_arttext\&pid=S1870-00632013000300011\&lng=es\&tlng=es.

19. González G, Vega M, Flores M. El incremento de la mortalidad por armas de fuego y su relación con el estancamiento de la esperanza de vida en México. Ciênc saúde coletiva [Internet]. 2017 [citado el 9 de enero de 2020]; 22 (9):2861-72. Disponible en: https://doi.org/10.1590/141381232017229.21902016 .

20. Martínez V, Teherán A, Cárdenas W, León L, Pimienta F, Barrera M, et al. Severidad del trauma calculada con registros de necropsia en diferentes tipos de lesión, Bogotá, 2013. Repositorio Institucional EdocUR [Internet]. 2018 [citado el 9 de enero de 2020]. Disponible en: https://repository. urosario.edu.co/handle/10336/18298?locale-attribute=pt.

21. Vega J, Gonzáles L. Determinar la frecuencia de muertes producidas por proyectil de arma de fuego en el SEMEFO de Iguala, Guerrero 2011 [tesis de licenciatura]. Toluca, Estado de México: Facultad de Medicina, Universidad Autónoma del Estado de México; 2014. Disponible en: http:// ri.uaemex.mx/handle/20.500.11799/14647.

22. Sankiti A, Guimarães A, Cavalcante R, Da Silva M, Cândido A, Batista P. Perfil epidemiológico das vítimas de morte violenta na Grande Cuiabá. Connect Line (Online) [Internet]. 2016 [citado el 11 de enero de 2020]; 15:118-31. Disponible en: http://periodicos.univag.com.br/index.php/ CONNECTIONLINE/article/view/354/580. 